\title{
Laboratory Diagnostics of Rickettsia Infections in Denmark 2008-2015
}

\author{
Susanne Schjørring ${ }^{1,2}$, Martin Tugwell Jepsen ${ }^{1,3}$, Camilla Adler Sørensen ${ }^{3,4}$, \\ Palle Valentiner-Branth ${ }^{5}$, Bjørn Kantsø ${ }^{4}$, Randi Føns Petersen ${ }^{1,4}$ (D), Ole Skovgaard ${ }^{6, *(D)}$ \\ and Karen A. Krogfelt 1,3,4,6,* \\ 1 Department of Bacteria, Parasites and Fungi, Statens Serum Institut (SSI), 2300 Copenhagen, Denmark; \\ ssc@ssi.dk (S.S.); martintjepsen@gmail.com (M.T.J.); rfp@ssi.dk (R.F.P.) \\ 2 European Program for Public Health Microbiology Training (EUPHEM), European Centre for Disease \\ Prevention and Control (ECDC), 27180 Solnar, Sweden \\ 3 Scandtick Innovation, Project Group, InterReg, 55111 Jönköping, Sweden; cas2300@gmail.com \\ 4 Virus and Microbiological Special Diagnostics, Statens Serum Institut (SSI), 2300 Copenhagen, Denmark; \\ bjoern@kantsoe.net \\ 5 Department of Infectious Disease Epidemiology and Prevention, Statens Serum Institut (SSI), \\ 2300 Copenhagen, Denmark; pvb@ssi.dk \\ 6 Department of Science and Environment, Roskilde University, 4000 Roskilde, Denmark \\ * Correspondence: olesk@ruc.dk (O.S.); karenak@ruc.dk (K.A.K.)
}

Received: 19 May 2020; Accepted: 15 June 2020; Published: 19 June 2020

\begin{abstract}
Rickettsiosis is a vector-borne disease caused by bacterial species in the genus Rickettsia. Ticks in Scandinavia are reported to be infected with Rickettsia, yet only a few Scandinavian human cases are described, and rickettsiosis is poorly understood. The aim of this study was to determine the prevalence of rickettsiosis in Denmark based on laboratory findings. We found that in the Danish individuals who tested positive for Rickettsia by serology, the majority $(86 \% ; 484 / 561)$ of the infections belonged to the spotted fever group. In contrast, we could confirm 13 of 41 (32\%) PCR-positive individuals by sequencing and identified all of these as $R$. africae, indicating infections after travel exposure. These 13 samples were collected from wound/skin material. In Denmark, approximately 85 individuals test positive for Rickettsia spp. annually, giving an estimated 26\% (561/2147) annual prevalence among those suspected of rickettsiosis after tick bites. However, without clinical data and a history of travel exposure, a true estimation of rickettsiosis acquired endemically by tick bites cannot be made. Therefore, we recommend that both clinical data and specific travel exposure be included in a surveillance system of Rickettsia infections.
\end{abstract}

Keywords: rickettsiosis; Rickettsia spp.; diagnostics of rickettsiosis; serology; PCR; tick-borne infections; vector-borne infections

\section{Introduction}

Tick-borne rickettsiosis is caused by obligate intracellular bacteria belonging to the genus Rickettsia and can be classified into two major disease groups: the spotted fever group (SFG), and the typhus group (TG). Rickettsia spp. are among the oldest known agents for vector-borne diseases, yet recently, an increase in incidence has been observed in the USA [1]. Variations in annual incidence may be attributed to a range of factors, such as human interaction with tick habitats, vector and host dynamics, climatic or ecological changes, increased awareness and testing of tick-borne diseases, or changes in surveillance practices [1]. 
Rickettsia spp. belonging to the SFG are often attributed to causing disease worldwide. The pathophysiology is characterized by invasion and replication in vascular endothelial cells, causing varying degrees of vasculitis in small to medium-sized blood vessels and resulting in symptoms such as fever, rash, headache, myalgia, arthralgia, and sometimes necrotizing eschar ("tache noir"). Clinical severity is often associated with the underlying species and ranges from potentially fatal diseases such as the Rocky Mountain spotted fever caused by Rickettsia rickettsii to the more benign African tick-bite fever caused by Rickettsia africae [1-3].

Until recently, the diagnosis of tick-borne SFG rickettsiosis was confirmed almost exclusively by serological methods because culturing needs specialized facilities. The oldest method for testing, the Weil-Felix test, is still used in developing countries [4,5]. This test is based on the detection of antibodies to formalin-inactivated whole cells of Proteus spp. (OX19, OX2, and OXK) that cross-react with Rickettsia of the SFG. However, this assay lacks sensitivity and specificity [6]. The microimmunofluorescence (MIF) assay is the current reference method for the detection of antibodies to Rickettsia spp., yet antigenic cross-reactions are seen within the SFG. Confirmation of tick-borne rickettsiosis in human samples has been supported and facilitated using molecular methods for identifying Rickettsia spp. A consensus on recommendations for the diagnosis of rickettsiosis was presented in "European guidelines for diagnosis of tick-borne diseases", which supports clinical diagnosis by molecular methods and serology [2].

In Denmark, only a limited number of studies have evaluated Rickettsia infections in humans after a tick bite. Furthermore, patients are rarely tested for Rickettsia after a tick bite, even though the presence of Rickettsia spp. in Denmark has been confirmed in ticks collected from domestic dogs or by flagging $[7,8]$. Rickettsia helvetica was found to be one of the most common pathogens in the Ixodes ricinus tick [7], but Anaplasma phagocytophilum, Borrelia burgdorferi, and R. helvetica have also been detected in I. ricinus ticks by PCR [9]. Previous studies have shown that Danish roe deer were seropositive for Borrelia spp., A. phagocytophilium, and tick-borne encephalitis virus (TBEV) [10]. Although there are several reports of ticks colonized by Rickettsia spp. [7-9] and of Rickettsia seropositivity in selected groups of humans [11-15], no systematic analysis of Rickettsia spp. prevalence and transmission has been conducted nationwide.

In one study, patients positive for B. burgdorferi were screened for Rickettsia antibodies, and $12.5 \%$ (21/168) were found positive for R. helvetica antibodies [11]. Despite a high frequency of tick bites, antibodies against Rickettsia were not detected in Danish elite orienteers [12]. Rickettsia has also been ruled out for involvement in the pathogenesis of sarcoidosis [13].

Rickettsiosis is potentially underestimated in Danish travelers returning from Africa, Southeastern Europe, and the US, where Rickettsia africae is the agent of African tick-bite fever (ATBF) and R. rickettsii and $R$. conori are agents of spotted fever group, respectively [6,14-17]. As rickettsiosis is not notifiable in Denmark, clinical and travel information is often lacking, which may cause trouble when trying to describe the disease prevalence. Furthermore, a recent study on the clinical assessment of rickettsiosis acquired endemically in Denmark suggests that the disease presents with mild symptoms compared with imported Rickettsia infections [3]. The quality and precision of national surveillance data depend on clinical and reporting practices for rickettsiosis.

In this study, we assessed samples submitted for routine diagnosis of rickettsiosis in Denmark in the period 2008-2015. Our aim is to enhance the awareness of rickettsiosis after tick bites and provide a basis for recommendations in managing tick-borne infections in Denmark.

\section{Materials and Methods}

\subsection{Data Collection and Study Population}

Data from all samples tested for Rickettsia spp. DNA and specific Rickettsia antibodies (IgG and IgM) from 1 July 2008 to 19 October 2015 for all of Denmark were retrieved from the laboratory database at Statens Serum Institut (SSI). Age, sex, sample date, as well as travel and clinical information (when provided) were recorded. Clinical and travel data were analyzed and presented by Ocias et al. [3]. 
The data analysis was performed on samples from individuals with a permanent address in Denmark, excluding Greenland. Statistics and conclusions summarized in this report represent descriptions of the cases captured in the reporting period of this study. In total, 2819 samples obtained from 2240 individuals during this period were analyzed (Figure 1).

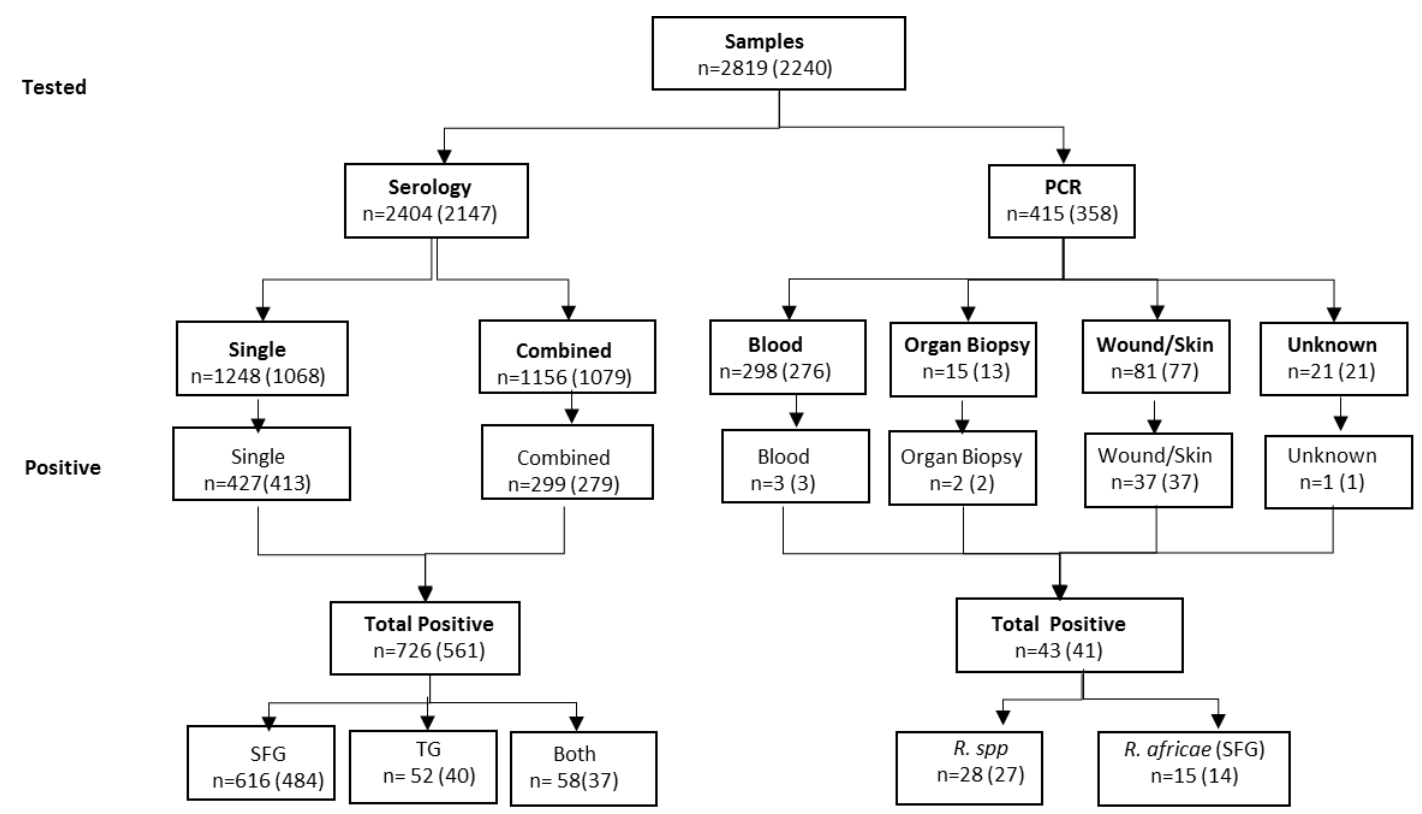

Figure 1. The number of tested samples and the number of positive samples detected by immunofluorescent assay (IFA) and PCR from 2008 to 2015. n: tested samples (number of patients).

\subsection{Rickettsia Antibody Detection}

Detection of IgG and IgM for SFG was performed using Focus Diagnostics' Immunofluorescent assay (IFA) (IF0100G/IF0100M) with antigens from the spotted fever group (Rickettsia rickettsii) and the typhus group (Rickettsia typhi). Positive cut-off values were $\operatorname{IgG} \geq 1: 512$ and/or $\operatorname{IgM} \geq 1: 64$ and adjusted to the Danish population and exposure [18].

Additionally, detection of IgG and IgM antibodies against Bartonella henselae and Bartonella quintana and IgG against Anaplasma phagocytophilum was performed as described by the manufacturer (Focus Diagnostics IFA IF1300G/IF1300M and IF1450G respectively). Detection of Francisella tularensis was performed by an in-house agglutination test with cut-off $\geq 1: 10$. The detection of $\operatorname{IgG}$ and IgM antibodies against $B$. burgdorferi was performed by an in-house ELISA modified from Hansen et al. [19].

\subsection{Detection of Rickettsia spp. DNA by PCR Assay and Sequencing}

Real-time PCR was used to amplify a 74-bp fragment of the citrate synthase (CS) gene (gltA) present in all Rickettsia spp., according to Stenos et al. [20]. DNA from plasma/serum samples were purified by QIAamp DNA Mini Kit (Qiagen 51,306) according to the manufacturer's instructions. Samples positive for gltA amplification were further analyzed by conventional 16S RNA sequencing using PCR primers (Rick16SF2 5' -ACG CTA TCG GTA TGC TTA ACA CAT G-3' and Rick16SR2 5' -CAA CTT ACT AAA CCG CCT ACG CAC T-3') targeting the $16 \mathrm{~S}$ rRNA gene for species identification. The sequences were compared to the Nucleotide collection (nr/nt) using NCBI BLAST. Positive control DNA was kindly provided by Professor John Stenos [20]. 


\section{Results}

\subsection{Diagnostic Samples}

In the years 2008-2015, a total of 2819 samples were analyzed with either PCR and/or serology testing (Table 1, Figure 1). The majority of samples were tested for the presence of Rickettsia antibodies $(2404,85 \%)$, and of these, 1156 (48\%) were tested for the presence of additional antibodies against four other tick-borne or related bacteria, namely, Bartonella, Anaplasma, Francisella, and Borrelia spp.

Table 1. Distribution of samples submitted to Statens Serum Institut and tested for rickettsiosis.

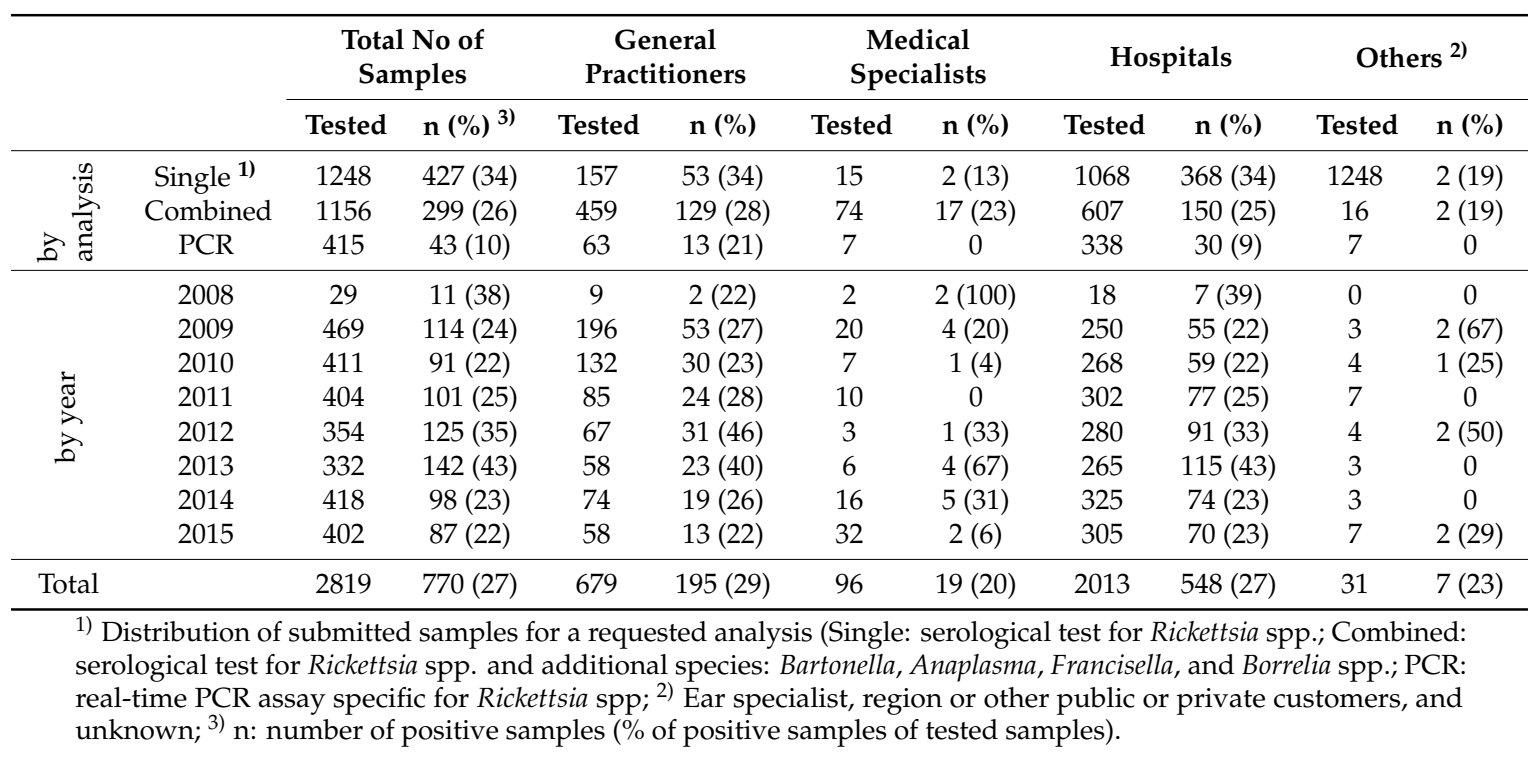

Real-time PCR analysis was applied to 415 (15\%) samples. These samples were categorized: blood (serum/plasma), unspecified organ biopsy, wound/skin, and unspecified (Figure 1). Most of the samples tested by PCR were serum/plasma (72\%; 298/415). Within the category of serum/plasma, $79 \%(236 / 298)$ were obtained in ethylenediaminetetraacetic acid (EDTA) tubes, where only three samples $(1 \% ; 3 / 235)$ tested positive by PCR. The positive rates for organ biopsy and wound/skin were $13 \%(2 / 15)$ and $46 \%(37 / 81)$, respectively (Figure 1$)$.

16S RNA sequencing was attempted on all samples that tested positive for Rickettsia gltA by real-time PCR $(n=43)$. Thirteen PCR-positive samples were sequenced successfully; the remaining 30 samples did not yield sequence templates of suitable quality. Twelve samples perfectly matched R. africae, acc. nos. CP001612 and L36098; the last sample had one mismatch with these sequences, but $R$. africae was still the closest match. Only 5 of these $13 R$. africae-positive patients had reported travel to Africa, while the rest did not provide travel information. The samples that could be sequenced successfully were all taken from wounds or skin biopsies. The obtained sequences are given in Supplementary Material.

In total, 769 samples tested positive for Rickettsia in the period 2008-2015 in Denmark; of these, $726(94 \%)$ were positive by serology and $43(6 \%)$ by PCR (Figure 1$)$.

\subsection{Samples Tested for Rickettsia Antibodies}

We found a similar distribution between samples positive for Rickettsia antibodies that were tested for Rickettsia antibodies only $(34 \%, 427 / 1248)$ and samples that were also analyzed for the presence of antibodies against other agents of tick-borne infections $(26 \%, 299 / 1156)$ (Figure 1).

However, the absolute antibody titers were, on average, higher in samples tested only for Rickettsia antibodies compared to those being tested for additional tick-borne infection (data not shown). Most of 
the investigated samples $(71 \%, 2013 / 2823)$ were collected in hospitals, primarily from departments of infectious diseases $(49 \% ; 1383 / 2823)$. Other departments included dermatology, neurology, and others.

\subsection{Age and Gender Distribution of Patients with Rickettsia Antibodies}

In the study period, 769 positive samples represent 591 patients with confirmed Rickettsia infection. Serology testing was the most common method used for the diagnosis of Rickettsia infections. Overall, the infected individuals were almost evenly distributed with regard to sex, as 51\% (299/591) of the patients were male (Figure 2). The age group $>30-60$ included 55\% (325/591) of the patients. The median age was 47 years (ranging from 1 year to 86 years). Children below the age of 18 had a lower positivity rate compared to adults.

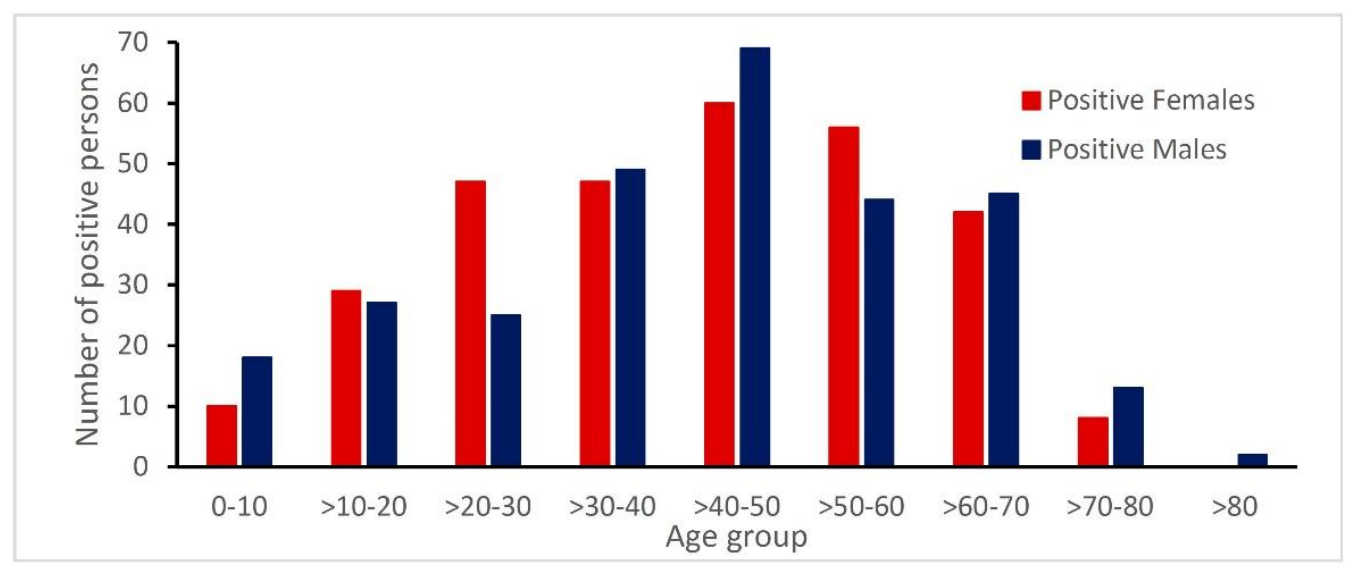

Figure 2. Age and gender distribution of confirmed Rickettsia-infected patients.

\subsection{Geographical Distribution of Danish Cases}

Accurate determination of the geographical distribution of cases within Denmark was difficult due to the large area that each hospital covers. Only 11\% (61/561) of the seropositive individuals had reports of foreign travel. Of the individuals found positive for rickettsiosis, only $5 \%$ (31/602) reported a previous tick bite.

\subsection{Seroprevalence of Rickettsia spp. in Denmark}

The serology test included IgG and IgM analysis for both SFG and TG (Table 2). Out of the 2404 serology tested samples, 726 samples were positive for either IgG and/or IgM, representing 561 individuals with antibodies against Rickettsia. Only 4\% (81/2075) of the patients had two subsequent serology samples tested 14-31 days apart, even though follow-up is highly recommended for serology.

Table 2. Distribution of the number of 561 individuals according to serology detection.

\begin{tabular}{ccccc}
\hline & \multicolumn{2}{c}{ SFG } & \multicolumn{2}{c}{ TG } \\
\cline { 2 - 5 } Positive & \multicolumn{2}{c}{ n (\%) } & \multicolumn{3}{c}{ n (\%) } \\
\cline { 2 - 5 } & $\mathbf{4 8 4}$ & $\mathbf{( 8 6 )}$ & $\mathbf{4 0}$ & (7) \\
\hline IgM & 401 & $(83)$ & 18 & $(46)$ \\
IgM and IgG & 44 & $(9)$ & 1 & \\
IgG & 39 & $(8)$ & 21 & $(54)$ \\
\hline
\end{tabular}

1) $\mathrm{n}$ : Number of positive individuals (\% of positive). Thirty-seven (7\%) individuals had antibodies to both SFG and TG (inconclusive). 


\subsection{Concordance Between PCR and Serology}

Overall, of the 358 individuals tested by PCR, only 193 were followed up with a serology test within 3 months of the PCR test. During this period, 119 individuals tested negative by both PCR and serology, but no follow-up sample was submitted within a 7-day period. Eight individuals were positive by PCR but were serologically negative; four of these were identified as $R$. africae infections. No follow up serology was done.

Fifty-five individuals tested positive by serology but were negative by PCR. In 14 individuals, PCR was performed from 4 days up to 4 months after the serological test. The unpaired samples for PCR and serology is a weakness for the correct diagnosis of rickettsial infections. Positive PCR tests are not followed up for antibodies and vice versa. Often, the serological testing is performed late in the course of the disease, and naturally, the PCR will be negative since the bacterium is not present.

\subsection{Co-Infections}

Out of the 2404 serology-tested samples, 1156 samples were tested for additional antibodies from relevant tick-borne infections. A total of 299 samples representing 279 individuals were positive for Rickettsia spp.; 10 of those individuals showed an immune response to at least two other species, indicating possible co-infections. Most co-infections were between Rickettsia spp. and Bartonella spp., or Rickettsia spp. and Borrelia spp. (Table 3).

Table 3. Co-infections with Rickettsia spp. and other tick-related infections ${ }^{\mathbf{1}) .}$

\begin{tabular}{|c|c|c|c|c|c|}
\hline No. of Co-Infections & Rickettsia (R) & $\begin{array}{c}\text { B. henselae } \\
\text { B. quintana }\end{array}$ & B. burgdorferi ${ }^{2)}$ & Ehrlichia ${ }^{3)}$ & F. tularensis ${ }^{3)}$ \\
\hline 0 & 197 & & & & \\
\hline 1 & 72 & 17 & 32 & 15 & 8 \\
\hline 2 & 4 & 4 & 4 & & \\
\hline 2 & 3 & & 3 & 3 & \\
\hline 2 & 1 & 1 & & 1 & \\
\hline 2 & 1 & & & 1 & 1 \\
\hline 2 & 1 & & 1 & & 1 \\
\hline Total & 279 & 22 & 40 & 20 & 10 \\
\hline
\end{tabular}

1) Two-hundred-seventy-nine individuals who tested positive for Rickettsia spp. (IgG and/or IgM) were combination tested for Bartonella, Ehrlichia, F. tulearensis, and B. burgdorferi antibodies. ${ }^{2)}$ Tested with IgG and/or IgM. ${ }^{3)}$ Tested with IgG only.

\section{Discussion}

Scandinavian ticks are reported to be infected with Rickettsia, yet only a few Scandinavian human case reports of rickettsiosis are published in the scientific literature $[6,11,14,21]$. A report from the European Centre for Disease Prevention and Control (ECDC) concludes that rickettsiosis is poorly understood but is considered an emerging disease in Europe, and reliable data on disease risk are lacking. The report also concludes that it is not possible to prioritize rickettsiosis over other emerging diseases [16].

Accordingly, improved surveillance of rickettsiosis throughout Europe is necessary. Denmark does not have a surveillance program for rickettsiosis, and SSI is the only laboratory in Denmark that tests for human Rickettsia infections. Therefore, this retrospective study is nationwide and elucidates the number of Danish Rickettsia infections and the distribution by age and gender, together with estimates of co-infections.

In this study, we found an average seroprevalence of $26 \%$ (561/2147 seropositive samples: Figure 1$)$ for Rickettsia antibodies in the study period from 2008 to 2015. In the interpretation of the serological tests, the positive cut-off value was adjusted with respect to the Danish background antibody level in a healthy population [18]. In this study, very few (approximately $4 \%$; data not shown) of the individuals had repeated serology testing between 14 and 21 days later, which emphasizes the need to stress the importance of correct diagnosis to medical doctors. A confirmed case of acute rickettsiosis detected 
by serology requires seroconversion testing in a second sample, with approx. $2-3$ weeks between samplings [17].

Analysis for the presence of Rickettsia DNA by PCR and sequencing revealed that skin samples and biopsies were superior to blood samples. Our results confirm previously described findings of low PCR sensitivity in serum samples [22], as just 6\% (43/726) of the positive samples were found by PCR.

There is an emerging consensus that ticks may carry several pathogens (see [7] for a recent Danish study), but there are only a few available reports on co-infections in humans acquired from tick vectors. In one Polish study, 110 patients with tick-borne encephalitis (TBE) were analyzed for coinfections, and the authors found 30 and 12 PCR-positive patients for Borrelia spp. and A. phagocytophilum, respectively [23]. In another Polish study, a single case of A. phagocytophilum and a single case of Babesia spp. co-infections were found in 24 Borrelia spp.-positive samples [24]. In a Danish study, 168 patients tested positive for antibodies against $R$. helvetica; the study found that 21 patients (12.5\%) also had antibodies against $R$. helvetica [11].

In the present study, indications of co-infections of Rickettsia with either Borrelia spp. (14\%; 40/279) or Bartonella spp. ( $8 \%$ 22/279) were found. Others have reported that co-infections of Babesia spp. and Borrelia spp. resulted in prolonged and more intense clinical manifestations than those resulting from infection with Borrelia alone [25]. It is possible that the variety of clinical symptoms and outcomes in patients highly depends on the presence of co-infections.

Our data show that hospitals have submitted more samples for Rickettsia testing than general practitioners. This indicates a greater degree of rickettsiosis awareness among hospital personnel or that the patients arriving at the hospital have severe and clear symptoms indicative of a need for testing. It could also reflect the possibility that patients visiting general practitioners present with fewer/milder symptoms, or they are in the early stages of the disease.

Tick-borne rickettsiosis (due to the wide range of symptoms) can be mistaken for medical conditions such as viral gastroenteritis, upper respiratory tract infection, non-Rickettsia bacterial sepsis, idiopathic vasculitis, and viral or bacterial meningoencephalitis [26,27]. Despite non-specific initial symptoms of tick-borne rickettsiosis (e.g., fever, malaise, and headache), early consideration in the differential diagnosis and empiric treatment is critical to preventing poor outcomes, especially for Rocky Mountain spotted fever, which progresses rapidly without treatment [3]. The dermatologic classification of the rash, its distribution, a pattern of progression and timing relative to the onset of fever, and other systemic signs provide clues to help guide the differential diagnosis [17]. Recent reports from Asia and the Mediterranean region show that Rickettsia species (such as $R$. acarii and $R$ felis) can also be transmitted by mites, fleas, and mosquitos [28-30]. Description of the rash after a tick bite or other insect bite, supported by the timely collection of samples for PCR and serology, will ensure the correct diagnosis of vector-borne infections. A limitation of the study is that sparse information regarding both the clinical condition and travel exposure was given along with the patient information. Clinical information could allow for correlation with diagnostic tests and calculation of known risk factors. Information on travel exposure would also make it possible to calculate a correct estimate of Rickettsia infections acquired in Denmark by excluding all travel-related infections. It is, therefore, important to be informed about travel and the clinical picture of the individual [3].

The present retrospective study from Denmark on laboratory evidence for SFG infections underlines the importance of alertness and diagnostic preparedness for adequate handling of rickettsial infections.

\section{Conclusions}

In conclusion, seropositivity of $26 \%$ for Rickettsia antibodies was observed in Danish individuals suspected of tick-borne disease. This high prevalence suggests that Rickettsia is endemic in Denmark, and, as previously described, patients are presenting with mild symptoms. The test can be used in a differential diagnosis for other vector-borne infections. Improved utilization of appropriate diagnostic 
tests, documentation of epidemiological factors, and timely reporting to public health officials will guide prevention messaging and shape public health policies.

We will stress the need for increased public awareness and strengthening of Rickettsia surveillance, as Rickettsia infections could result in an increasing disease burden as people travel more, and concerns about an increasing number of immunocompromised individuals are rising. Therefore, we recommend that both clinical data and specific travel exposure be included in the surveillance system of Rickettsia infections.

Supplementary Materials: The following are available online at http://www.mdpi.com/2079-7737/9/6/133/s1, Partial 16S RNA sequences obtained from samples that tested positive for Rickettsia gltA by real-time PCR.

Author Contributions: Conceptualization, K.A.K. and P.V.-B.; Methodology, S.S., B.K., C.A.S., and R.F.P.; Software, S.S.; Validation, B.K. and K.A.K.; Formal analysis, S.S. and P.V.-B.; Investigation, K.A.K.; Resources, K.A.K.; Data curation, S.S. and P.V.-B.; Writing—original draft preparation, B.K., P.V.-B., S.S., R.F.P., and K.A.K.; Writing - review and editing, K.A.K., M.T.J., and O.S., Supervision, K.A.K.; Project administration, K.A.K.; Funding acquisition, K.A.K. All authors have read and agreed to the published version of the manuscript.

Funding: The study was partly funded by the InterReg V Program (the Scandtick Innovation project, grant number 20200422). The sponsor was not involved in the design and execution of the study, analysis of data, or the writing and submission of the manuscript.

Acknowledgments: We would like to thank Mona Skafte Rønkendorff, Maja Olsen, Hanne Tove Andersen and Gitte I. Jensen at the Department of Virus and Microbiological Special Diagnostics, SSI, for technical assistance. We thank Charlotte Sværke Jørgensen, Head of the serology laboratory, SSI and Lukas Ocias, MD, SSI for critical discussions and suggestions throughout the project. Furthermore, we wish to thank EUPHEM framework programme and Head of Programme Aftab Jasir for epidemiological suggestions. Research involved in North Sea Region programme NorthTick, (Grant no. J-No.: 38-2-7-19) are thanked for inspiring discussions about ticks and tick-borne infections.

Conflicts of Interest: The authors declare no conflict of interest. The funders had no role in the design of the study; in the collection, analyses, or interpretation of data; in the writing of the manuscript, or in the decision to publish the results.

\section{References}

1. Drexler, N.A.; Dahlgren, F.S.; Massung, R.F.; Behravesh, C.B.; Heitman, K.N.; Paddock, C.D. National surveillance of spotted fever group rickettsioses in the United States, 2008-2012. Am. J. Trop. Med. Hyg. 2016, 94, 26-34. [CrossRef] [PubMed]

2. Brouqui, P.; Bacellar, F.; Baranton, G.; Birtles, R.; Bjöersdorff, A.; Blanco, J.-R.; Caruso, G.; Cińco, M.; Fournier, P.; Francavilla, E.; et al. Guidelines for the diagnosis of tick-borne bacterial diseases in Europe. Clin. Microbiol. Infect. 2004, 10, 1108-1132. [CrossRef] [PubMed]

3. Ocias, L.F.; Jensen, B.B.; Villumsen, S.; Lebech, A.-M.; Skarphedinsson, S.; Dessau, R.B.; Krogfelt, K. Rickettsioses in Denmark: A retrospective survey of clinical features and travel history. Ticks Tick Borne Dis. 2018, 9, 573-579. [CrossRef]

4. Kularatne, S.A.M.; Rajapakse, R.; Wickramasinghe, W.; Nanayakkara, D.; Budagoda, S.; Weerakoon, K.G.; Edirisinghe, J.; Premaratna, R. Rickettsioses in the central hills of Sri Lanka: Serological evidence of increasing burden of spotted fever group. Int. J. Infect. Dis. 2013, 17, e988-e992. [CrossRef] [PubMed]

5. Kularatne, S.A.M.; Gawarammana, I. Validity of the Weil-Felix test in the diagnosis of acute rickettsial infections in Sri Lanka. Trans. R. Soc. Trop. Med. Hyg. 2009, 103, 423-424. [CrossRef]

6. Madsen, K.M.; Storgaard, M.; Krogfelt, K.A.; Obel, N. Rickettsiosis after a stay in South Africa. Ugeskr. Laeger 2004, 166, 902-904.

7. Stensvold, C.R.; Al Marai, D.; Andersen, L.O.; Krogfelt, K.; Jensen, J.S.; Larsen, K.S.; Nielsen, H. Babesia spp. and other pathogens in ticks recovered from domestic dogs in Denmark. Parasites Vectors 2015, 8, 262. [CrossRef]

8. Kantsø, B.; Svendsen, C.B.; Jensen, P.M.; Vennestrøm, J.; Krogfelt, K. Seasonal and habitat variation in the prevalence of Rickettsia helvetica in Ixodes ricinus ticks from Denmark. Ticks Tick Borne Dis. 2010, 1, 101-103. [CrossRef] 
9. Skarphedinsson, S.; Lyholm, B.F.; Ljungberg, M.; Søgaard, P.; Kolmos, H.J.; Nielsen, L.P. Detection and identification of Anaplasma phagocytophilum, Borrelia burgdorferi, and Rickettsia helvetica in Danish Ixodes ricinus ticks. APMIS 2007, 115, 225-230. [CrossRef]

10. Skarphedinsson, S.; Jensen, P.M.; Kristiansen, K. Survey of tickborne infections in Denmark. Emerg. Infect. Dis. 2005, 11, 1055-1061. [CrossRef]

11. Nielsen, H.; Fournier, P.-E.; Pedersen, I.S.; Krarup, H.; Ejlertsen, T.; Raoult, D. Serological and molecular evidence of Rickettsia helvetica in Denmark. Scand. J. Infect. Dis. 2004, 36, 559-563. [CrossRef] [PubMed]

12. Schiellerup, P.; Dyhr, T.; Rolain, J.-M.; Christensen, M.; Damsgaard, R.; Fisker, N.; Andersen, N.F.; Raoult, D.; Krogfelt, K. No serological evidence for Rickettsial diseases among Danish elite Orienteerers. Ann. N. Y. Acad. Sci. 2006, 1078, 150-153. [CrossRef]

13. Svendsen, C.B.; Milman, N.; Dziegiel, M.H.; Krogfelt, K.; Høier-Madsen, M. Determination of rickettsial and antinuclear antibodies in Danish patients with sarcoidosis. Clin. Respir. J. 2008, 2, 202-207. [CrossRef] [PubMed]

14. Johansen, J.A.; Thybo, S. African Tick Bite Fever upon game hunting in South Africa. Ugeskr. Laeger 2011, 173, 2572-2573.

15. Kibsgaard, L.; Lindberg, J.; Villumsen, S.; Larsen, C.S. Rickettsiosis should be considered as a differential diagnosis in patients having fever related to travelling. Ugeskr. Laeger 2012, 174, 1525-1528. [PubMed]

16. ECDC. Epidemiological Situation of Rickettsioses in EU/EFTA Countries; European Centre for Disease Prevention and Control: Stockholm, Sweden, 2013. [CrossRef]

17. Biggs, H.M.; Behravesh, C.B.; Bradley, K.K.; Dahlgren, F.S.; Drexler, N.A.; Dumler, J.S.; Folk, S.M.; Kato, C.Y.; Lash, R.R.; Levin, M.L.; et al. Diagnosis and management of Tickborne Rickettsial diseases: Rocky mountain spotted fever and other spotted fever group Rickettsioses, Ehrlichioses, and Anaplasmosis-United States. MMWR Recomm. Rep. 2016, 65, 1-44. [CrossRef]

18. Kantsø, B.; Svendsen, C.B.; Jørgensen, C.S.; Krogfelt, K. Evaluation of serological tests for the diagnosis of rickettsiosis in Denmark. J. Microbiol. Methods 2009, 76, 285-288. [CrossRef]

19. Hansen, K.; Hindersson, P.; Pedersen, N.S. Measurement of antibodies to the Borrelia burgdorferi flagellum improves serodiagnosis in Lyme disease. J. Clin. Microbiol. 1988, 26, 338-346. [CrossRef]

20. Stenos, J.; Unsworth, N.B.; Graves, S.R. A highly sensitive and specific real-time PCR assay for the detection of spotted fever and typhus group Rickettsiae. Am. J. Trop. Med. Hyg. 2005, 73, 1083-1085. [CrossRef]

21. Nilsson, K.; Elfving, K.; Påhlson, C. Rickettsia helveticain Patient with Meningitis, Sweden, 2006. Emerg. Infect. Dis. 2010, 16, 490-492. [CrossRef]

22. Wood, H.; Artsob, H. Spotted fever group Rickettsiae: A brief review and a Canadian perspective. Zoonoses Public Health 2012, 59, 65-79. [CrossRef]

23. Moniuszko-Malinowska, A.; Dunaj, J.; Swiecicka, I.; Zambrowski, G.; Chmielewska-Badora, J.; Żukiewicz-Sobczak, W.; Zajkowska, J.; Czupryna, P.; Kondrusik, M.; Grygorczuk, S.; et al. Co-infections with Borrelia species, Anaplasma phagocytophilum and Babesia spp. in patients with tick-borne encephalitis. Eur. J. Clin. Microbiol. Infect. Dis. 2014, 33, 1835-1841. [CrossRef] [PubMed]

24. Welc-Falęciak, R.; Hildebrandt, A.; Siński, E. Co-infection with Borrelia species and other tick-borne pathogens in humans: Two cases from Poland. Ann. Agric. Environ. Med. 2010, 17, 309-313. [PubMed]

25. Krause, P.J.; McKay, K.; Thompson, C.A.; Sikand, V.K.; Lentz, R.; Lepore, T.; Closter, L.; Christianson, D.; Telford, S.R.; Persing, D.; et al. Disease-specific diagnosis of Coinfecting Tickborne Zoonoses: Babesiosis, human granulocytic Ehrlichiosis, and lyme disease. Clin. Infect. Dis. 2002, 34, 1184-1191. [CrossRef]

26. Dumler, J.S.; Walker, D.H. Ehrlichia chaffeensis (human Monocytotropic Ehrlichiosis), Anaplasma phagocytophilum (human Granulocytotropic Anaplasmosis), and other Anaplasmataceae. In Mandell, Douglas, and Bennett's Principles and Practice of Infectious Diseases; Elsevier Inc.: Philadelphia, PA, USA, 2014; Volume 2, pp. 2227-2233. [CrossRef]

27. Walker, D.; Blanton, L. Rickettsia rickettsii and other spotted fever group Rickettsiae (Rocky Mountain spotted fever and other spotted fevers). In Mandell, Douglas, and Bennett's Principles and Practice of Infectious Diseases; Elsevier Inc.: Philadelphia, PA, USA, 2014; Volume 2, pp. 2198-2205. [CrossRef]

28. Robinson, M.T.; Satjanadumrong, J.; Hughes, T.; Stenos, J.; Blacksell, S.D. Diagnosis of spotted fever group Rickettsia infections: The Asian perspective. Epidemiol. Infect. 2019, 147, e286. [CrossRef] [PubMed] 
29. Adem, P.V. Emerging and re-emerging rickettsial infections. Semin. Diagn. Pathol. 2019, 36, $146-151$. [CrossRef] [PubMed]

30. Charrel, R.N.; Berenger, J.-M.; Laroche, M.; Ayhan, N.; Bitam, I.; Delaunay, P.; Parola, P. Neglected vector-borne bacterial diseases and arboviruses in the Mediterranean area. New Microbes New Infect. 2018, 26, S31-S36. [CrossRef] 